- There was no link between nerve damage and anaesthetic type.

- Third molar uneruption was a predictor for nerve injury.

- Retraction of lingual nerve was strongly associated with nerve damage.

- There was no association between age of the patient and nerve damage.

- Seniority of the operator had no effect on the morbidity of the both lingual and IDN.

\title{
Links between anaesthetic modality and nerve damage during lower third molar surgery
}

\author{
K. Rehman, ${ }^{1}$ K. Webster ${ }^{2}$ and M. S. Dover ${ }^{3}$
}

Objective To investigate the relationships between eruption status, gender, social class, grade of operator, anaesthetic modality and nerve damage during third molar surgery.

Design Two centre prospective longitudinal study.

Setting The department of oral and maxillofacial surgery, University Hospital Birmingham NHS Trust and oral surgery outpatient clinics at Birmingham Dental Hospital.

Subjects A total of 391 patients had surgical removal of lower third molars. Sensory disturbance was recorded at one week post operatively. Patients with altered sensation were followed up at one month, three months and six months following surgery.

Results 614 lower third molars in 391 patients were removed. Fortysix procedures (7.5\%) were associated with altered sensation at one week with three procedures $(0.49 \%)$ showing persistent symptoms at six months. Of these 46 nerve injuries, 26 (4.23\%) involved the lingual nerve and 20 (3.25\%) the inferior dental nerve (IDN). All three persistent sensations were IDN related. A logistic regression model found that the use of a lingual retractor $\chi^{2}=11.559, p=0.003$ was more significant than eruption status $\chi^{2}=12.935, p=0.007$. There was no significant relationship between anaesthetic modality, age, social class, sex and seniority of operator.

Conclusions There was no link between the choices of local or general anaesthesia and nerve damage during lower third molar removal when difficulty of surgery was taken into account.

Third molar surgery may be associated with iatrogenic nerve damage. The incidence of nerve damage following the removal of third molars is well documented. Lingual nerve injury ranges from $0.5 \%-22 \%$ and inferior dental nerve (IDN) injury between $0.5 \%-7 \%$ with a mean of $4 \% .{ }^{1-5}$ Several factors have been associated with an increased risk of nerve damage; the removal of unerupted teeth, ${ }^{6}$ lingual retraction using a narrow instrument

${ }^{1 *}$ K. Rehman, Medical Student, University of Birmingham, ${ }^{2}$ K. Webster, Consultant Maxillofacial Surgeon, University Hospital Birmingham NHS Trust/ Birmingham Dental School, Department of Oral \&t Maxillofacial Surgery ${ }^{3}$ M. S. Dover, Consultant Maxillofacial Surgeon, University Hospital Birmingham NHS Trust/ Birmingham Dental School, Department of Oral \& Maxillofacial Surgery

*Correspondence to: K. Rehman, 15 Chaple Street, Lye, Stourbridge, West Midlands DY9 8BT

\section{Refereed paper}

Received 12.01.01; Accepted 20.03.02

๑ British Dental Journal 2002; 192: 43-45 such as a Howarth's, ${ }^{5-7}$ (although use of a broader retractor (eg Hovell) may results in a decreased incidence), ${ }^{8,9}$ seniority of the operator ${ }^{10}$ and finally removal of teeth under general anaesthetic. ${ }^{3}$

Sensory disturbance can be a major source of patient dissatisfaction and this has partly led to the release of guidelines on the management of impacted third molars in order to reduce morbidity. The National Institute for Clinical Excellence (NICE) have released guidance on the indications for the removal of wisdom teeth. ${ }^{11}$ They state that the prophylactic removal of pathologyfree impacted third molars should be discontinued in the NHS. However the Scottish Intercollegiate Guideline Network (SIGN) leans towards a greater acceptance of prophylactic removal, although they note the conflicting evidence as to the most appropriate form of protection for the lingual nerve. ${ }^{12}$

A recent paper looking at factors influencing nerve damage during lower third molar surgery suggested that nerve damage was five times more frequent when performed under general rather than local anaesthesia. ${ }^{3}$ This study used the WHARFE score as an assessment of surgical difficulty, but this scoring system has not been validated. ${ }^{13}$ Our study also used a simple visual analogue score (VAS) to assess surgical difficulty.

The study was designed to investigate the hypothesis that the type of anaesthetic used has no effect as a risk factor for nerve injury.

\section{METHODS}

A consecutive series of patients referred for surgical removal of lower wisdom teeth over a period of ten months from September 1999 to June 2000 were included. At the initial consultation, the information collected included: age, gender, post code (social class using Townsend Bands), presenting complaint, state of eruption and presence of caries in the adjacent teeth.

Surgical difficulty was assessed using a panoramic radiograph (OPG) and a method described by MacGregor. This method rates surgical difficulty with a score between $0-16$. The scoring system uses: Winter's classification, ${ }^{14}$ height of the mandible, angle of the second molar, root shape and development of the third molar, follicle size and exit path of the third molar, (WHARFE). The same observer (KR) calculated the scores. Two consultant maxillofacial surgeons blinded to the anaesthetic modality used in surgery recorded a second visual analogue score (VAS) scoring surgical difficulty from the OPG alone between 0-10. 
The type of surgical procedure used for extraction of the teeth depended on state of eruption. All fully and partially erupted teeth were removed by simple elevation or by a distal relieving incision and elevation respectively. Those requiring a minor surgical procedure had a standard buccal mucoperiostal flap raised with or without lingual retraction. The retractors used were either a Howarth's or a Hovell, and their use was entirely the choice of the operating surgeon. Many of the procedures required buccal bone removal using a surgical bur before delivery of the tooth and wound closure was achieved with the use of resorbable sutures. All the patients were reviewed a week post operatively by the same observer to assess any loss of sensation in relation to lingual, IDN and buccal nerves. The sensory assessment and anatomical distribution was made using a sharp probe and cotton wool. This method was chosen as the degree of sensory deficit was not the focus of the study. Any positive results were recorded and these patients were seen again a month later, at three months and six months following surgery.

\section{RESULTS}

A total of 391 patients (614 teeth) were treated in this study. Two hundred and seventy three patients (474 teeth) were treated under general anaesthetic (GA) of which 201 (73.6\%) had bilateral extractions. Another 90 patients (105 teeth) were treated under LA, of which 15 (16.7\%) had bilateral extractions and 28 (35 teeth) had LA and IV sedation. Of the teeth extracted under GA 112 (23.6\%) were unerupted compared with 5 (3.6\%) under LA and LA/sedation, (Figs 1 and 2). The mean age was $30.3(\mathrm{SD}=10.58$, range $=16-52)$. A total of $24 \%$ of patients were of social class TB 1, 13\% TB 2, 20\% TB 3, 20\% TB 4 and 23\% TB 5. The mean WHARFE score for all the third molars was 5.4 and a mean VAS for teeth of 5 . There was no difference in surgical difficulty between GA (WS=5.8, VAS=5) and LA (WS=5.7, VAS=4.8). Junior staff, specialist registrars 193 (31.4\%), staff grades $68(11.1 \%)$ and senior house officers 285 (46.4\%) undertook the majority of the procedures, with consultants undertaking 68 (11.1\%) (Fig. 3). Retraction of the lingual nerve was undertaken in $340(71.7 \%)$ of GA cases compared with $45(32.1 \%)$ of LA cases.

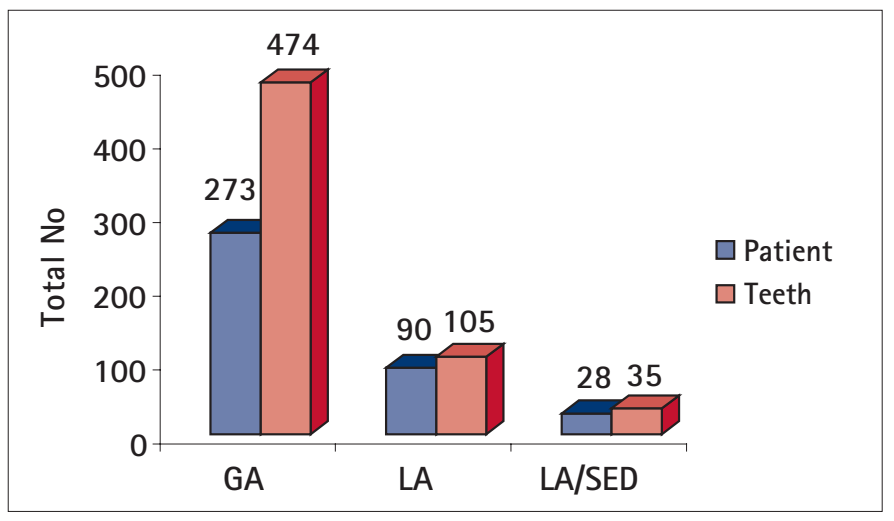

Figure 1 Number of patient and procedures

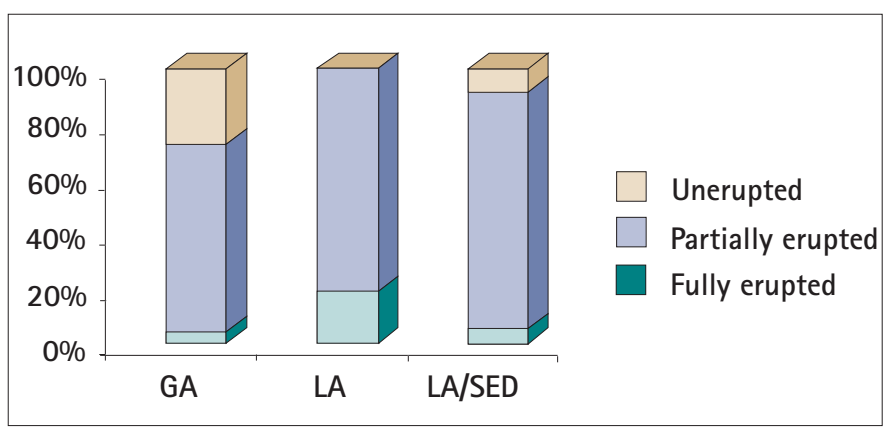

Figure 2 State of eruption
Forty-six procedures were associated with sensory loss, 26 (4.2\%) of which involved the lingual nerve and 20 (3.25\%) affecting the inferior dental nerve, (Table 1). This number reduced to 20 (3.25\%) lingual nerve and $10(1.63 \%)$ IDN, at one month, $2(0.32 \%)$ lingual nerve and $3(0.49 \%)$ IDN at three months and $3(0.49 \%)$ IDN at six months. A lingual retractor had been used in all those procedures where nerve damage was seen at one month review.

Table 1 Anaesthetic modality and nerve damage
\begin{tabular}{lccc}
\multicolumn{4}{c}{ Nerve damage } \\
\hline Anaesthetic modality & Number of teeth & Lingual & IDN \\
\hline Local anaesthetic & $105(17.1 \%)$ & $4(0.65 \%)$ & $5(0.8 \%)$ \\
Local anaesthetic/sedation & $35(5.7 \%)$ & 0 & 0 \\
General anaesthetic & $474(77.2 \%)$ & $22(3.58 \%)$ & $15(2.44 \%)$ \\
Total & $614(100 \%)$ & $26(4.23 \%)$ & $20(3.26 \%)$
\end{tabular}

Statistical analyses first using a univariate model of all factors recorded and nerve damage (lingual and IDN) revealed significant results associated with state of eruption; unerupted teeth were more likely to be associated with nerve damage $\left(\chi^{2}=12.935\right.$, $\mathrm{p}=0.003)$. The use of lingual retraction also significantly increased the risk of nerve injury $\left(\chi^{2}=11.759 p=0.007\right)$. There was no association of nerve damage with GA $\left(\chi^{2}=2.53, p=0.606\right)$, age was calculated to ten year banding $\left(\chi^{2}=16.28, p=0.234\right)$, pericoronitis $\left(\chi^{2}=5.75, p=0.062\right)$, caries of second molar $\left(\chi^{2}=0.662\right.$, $\mathrm{p}=0.732)$, operator seniority $\left(\chi^{2}=4.78, \mathrm{p}=0.559\right)$, cystic changes associated with wisdom teeth $\left(\chi^{2}=0.132, p=1.0\right)$ and sex of the patient $\left(\chi^{2}=0.39, p=0.8410\right)$ (Table 2$)$.

\begin{tabular}{lll}
\multicolumn{2}{l}{ Table 2 Statistical analyses } \\
\hline Factors & $\chi^{2}$ & p value \\
\hline GA v LA & 2.53 & 0.602 \\
Age & 16.28 & 0.234 \\
Pericoronitis & 5.75 & 0.062 \\
Caries & 0.66 & 0.732 \\
UE & 12.93 & 0.007 \\
Cystic change & 0.14 & 1 \\
Lingual Retraction & 11.76 & 0.003 \\
Operator & 4.78 & 0.559 \\
Sex & 0.39 & 0.841
\end{tabular}

However, when all the significant results were placed in a multivariate logistic regression model to find out which were more important, lingual retraction was of higher significance than state of eruption (Table 3).

\begin{tabular}{lccc}
\multicolumn{4}{l}{ Table 3 Logistic Regression Model } \\
\hline Factors & -2 Log likelihood & Cox \& Snell R Square & Nagelkerke R Square \\
\hline Eruption & 303.429 & .26 & .503 \\
Lingual retraction & 296.433 & .47 & .613
\end{tabular}

\section{DISCUSSION}

The results of this study have found no significant relationship between nerve damage and age, social class, the presence of pericoronitis, caries of adjacent teeth, anaesthetic modality and seniority of the operator. The relative risk of nerve damage was higher for the consultants than junior staff but was not significant $\left(\chi^{2}=4.78, p=0.559\right)$ These findings were inconsistent with those of Mason and Blackburn where senior staff had a much higher rate of nerve damage. ${ }^{6,15}$

The association between teeth being unerupted and an increased risk of nerve damage is shown in this study as confirmed by others. ${ }^{2,6,15}$ Over $90 \%$ of the unerupted teeth had lingual nerve retraction and the increased use of retraction in this group may explain the increased risk of nerve damage in these cases. Higher incidence of lingual nerve damage with use of lingual retractor is well documented and this, along with unerup- 


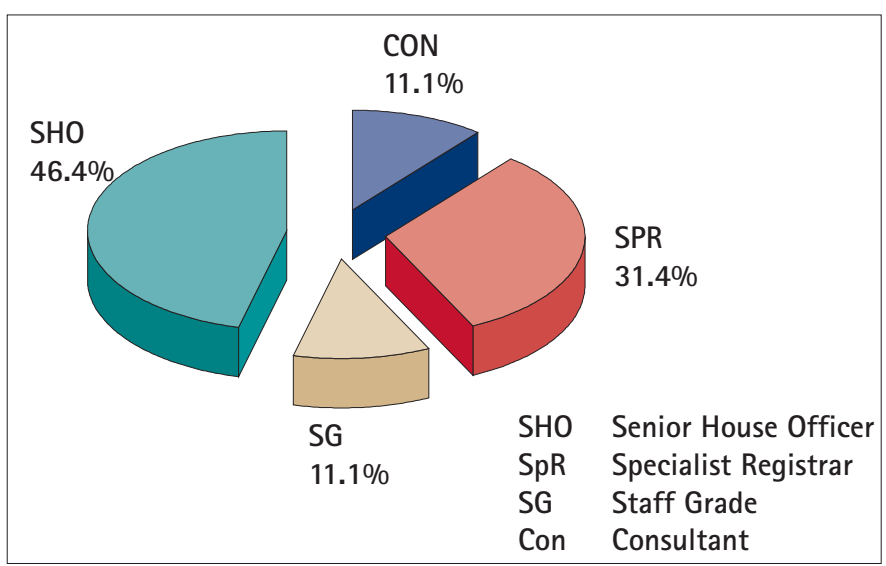

Figure 3 Seniority of the operator

tion status, probably accounts for an increased risk of nerve damage under GA rather than those cases performed under LA as suggested in other studies. ${ }^{3,16}$

The factors suggested by other authors leading to an increased rate of lingual nerve damage occurring following its retraction include anatomical variation of the lingual nerve, poor surgical technique and type of retractor used. An anatomical study by Behnia et al. revealed that in 94 cases (14.05\%), the nerve was above the lingual crest, and in 1 case $(0.15 \%)$, the nerve was in the retromolar pad region. ${ }^{17}$ The key to lingual retraction may be in creating 'tissue freedom' before insertion of a retractor, to avoid unnecessary stretching of the nerve. This theory expanded by Moss et al. suggests extending the lingual flap to the distal side of second molar to allow the insertion of a wider retractor, a technique which has shown a lower incidence rate in a single operator series. ${ }^{9}$ However, Robinson et al. have showed better results with avoidance of lingual retraction ${ }^{5}$ and a lower incidence of nerve injury in a group of dental students who changed from using a lingual retractor to no lingual retractor. ${ }^{4}$

The degree of surgical difficulty in both GA and LA groups was similar in this study, however an increase in incidence of nerve damage was associated with increase in WHARFE and VAS scores in this study. This shows that a VAS is as reliable and less complicated than other scoring systems in determining the surgical difficulty of removal of wisdom teeth. It has added benefits of taking into account the relationship of the inferior dental nerve to the tooth and predicting other complication such as fractured mandible in thin jaws, which MacGregor's WHARFE system does not take into account.

This study has not found any significant difference in morbidity of lingual or ID nerves for procedures performed under GA than LA. These findings are consistent with a more recent study by Hill et al. ${ }^{18}$ The paper by Brann et al. ${ }^{3}$ however suggested an increased risk of nerve injury for those wisdom teeth removed under GA. This study shows no such difference. Brann postulated that the degree of surgical force used was greater under GA than in a conscious patient. However our Wharfe and VAS scores were similar for cases performed under GA or LA and therefore there was no significant difference in surgical difficulty between these two groups.

In keeping with present guidelines the wisdom teeth removed in this study were symptomatic and therefore more likely to be partially erupted and subsequently less likely to be intimately related to the IDN.
In the United Kingdom the choice of LA or GA as an anaesthetic modality is normally a result of patient choice. There are many factors which predict choice of anaesthesia such as surgical difficulty, medical history of the patient and patient anxiety and a study by Edward et al. ${ }^{19}$ found that surgical difficulty was the most significant followed by patient anxiety, patients' preference and medical history. Our study was not aimed at looking into these factors but those which may be related to incidence of nerve damage. There is also no doubt that people having general anaesthetic suffer more post operative problems such as more time off work and hence increase the demand on the primary healthcare and these findings have been reported in the literature by Edward et al. ${ }^{20}$

This study does not support the proposal in the study by Brann et $a .^{3}$ that general anaesthesia is a risk factor for nerve injury. This study also suggests that a simple VAS is as useful in estimating difficulty.

The authors would like to thank all the staff at the Department of Maxillofacial Surgery, Selly Oak Hospital and Birmingham Dental Hospital particularly Helen Rafftry and the secretaries for their kind help. Many thanks also to Public Health and West Midlands Cancer Intelligence Unit for helping with statistics and social class data.

1. Gargallo-Albiol J, Buenechea-Imaz R, Gay-Escoda C. Lingual nerve protection during surgical removal of lower third molars. a prospective randomized study. Int J Oral Maxillofac Surg 2000 29: 268-71.

2. Gulicher D, Gerlach K L. [Incidence, risk factors and follow-up of sensation disorders after surgical wisdom tooth removal. Study of 1,106 cases]. Inzidenz, risikofaktoren und verlauf von sensibilitatsstorungen nach operativer weisheitszahnentfernung. Untersuchung an 1106 fallen. Mund Kiefer Gesichtschir 2000 4: 99-104.

3. Brann CR, Brickley M R, Shepherd J P. Factors influencing nerve damage during lower third molar surgery [see comments]. Br DentJ 1999; 186: 514-516.

4. Robinson P P, Loescher A R, Smith K G. The effect of surgical technique on lingual nerve damage during lower 3 rd molar removal by dental students. Eur J Dent Educ 1999; $3:$ :52-55

5. Robinson P P, Smith K G. Lingual nerve damage during lower third molar removal: a comparison of two surgical methods [see comments]. Br Dent J 1996; 180: 456-461.

6. Mason D A. Lingual nerve damage following lower third molar surgery. Int J Oral Maxillofac Surg 1988; 17: 290-294.

7. Smith K G, Robinson P P. An experimental study of three methods of lingual nerve defect repair. J Oral Maxillofac Surg 1995; 53: 1052-1062.

8. Greenwood M, Langton S G, Rood J P. A comparison of broad and narrow retractors for lingual nerve protection during lower third molar surgery. Br J Oral Maxillofac Surg 1994; 32: 114-117.

9. Moss CE, Wake M J. Lingual access for third molar surgery: a 20-year retrospective audit [see comments]. Br J Oral Maxillofac Surg 1999; 37: 255-258.

10. Venta I, Lindqvist C, Ylipaavalniemi P. Malpractice claims for permanent nerve injuries related to third molar removals. Acta Odontol Scand 1998; 56: 193-196.

11. National Institute for Clinical Excellence. Guidance on the removal of wisdom teeth. Technology appraisal guidance No1, 2000

12. Scottish Intercollegiate Guideline Network. Management of unerupted and impacted third molar teeth. A National Clinical Guideline. Evidence-Based Dentistry 2000; 2 44-45.

13. MacGregor A. The impacted lower wisdom tooth. Oxford; Oxford University Press: 1985: pp62.

14. Winter G B. Impacted mandibular third molars St Louis, American Medical Book Co.; 1926.

15. Blackburn CW, Bramley PA. Lingual nerve damage associated with the removal of lower third molars [see comments]. Br Dent J 1989; 167: 103-107.

16. Carmichael FA, McGowan D A. Incidence of nerve damage following third molar removal: a West of Scotland Oral Surgery Research Group study. Br J Oral Maxillofac Surg 1992; 30: 78-82.

17 Behnia $H$, Kheradvar A, Shahrokhi M. An anatomic study of the lingual nerve in the third molar region. J Oral Maxillofac Surg 2000; 58: 649-651; discussion 652-3.

18 Hill C M, Mostafa P. Thomas D W, Newcombe R G, Walker R V. Nerve morbidity following wisdom tooth removal under local and general anaesthesia. Br J Oral Maxillofac Surg 2001; 39: 419-422.

19 Edward D J, Brickley M R, Horton J, Edward M J, Shepherd J P. Choice of anaesthetic and health facility for third molar surgery. Br J Oral Maxillofac Surg 1998; 36: 333-340.

20 Edwards D J, Horton J, Shepherd J P, Brickley M R. Impact of third molar removal on demands for postoperative care and job disruption: does anaesthetic choice make a difference? Ann R Coll Surg Eng 1999 Mar; 81: 119-23. 